\title{
Acute Stroke Imaging in the Era of the DAWN, DEFUSE 3 and WAKE-UP Study Findings
}

\author{
Horst Urbach, ${ }^{1}$ Elias Kellner ${ }^{2}$ and Karl Egger ${ }^{1}$ \\ 1. Department of Neuroradiology, Medical Center and Faculty of Medicine, University of Freiburg, Freiburg, Germany; \\ 2. Department of Medical Physics, Medical Center and Faculty of Medicine, University of Freiburg, Freiburg, Germany
}

DOI: https://doi.org/10.17925/ENR.2019.14.1.24

$\mathrm{M}$ ost stroke centres perform multimodal computed tomography imaging in order to select patients with acute stroke for mechanical thrombectomy and/or intravenous (IV) thrombolysis. After the DAWN (Clinical Mismatch in the Triage of Wake up and Late Presenting Strokes Undergoing Neurointervention with Trevo Thrombectomy Procedure) and DEFUSE 3 (Endovascular Therapy Following Imaging Evaluation for Ischaemic Stroke 3) studies, the time window for the treatment of stroke has been extended from 6 hours to 24 hours provided that a small infarct core and significant tissue at risk is documented. After the WAKE-UP (Efficacy and Safety of MRI-based Thrombolysis in Wake-up Stroke) study, wake-up strokes can be selected for IV thrombolysis if diffusion-weighted imaging is positive and fluid-attenuated inversion recovery magnetic resonance imaging is negative. We propose an imaging algorithm in which the imaging modality is mainly selected with respect to the National Institutes of Health Stroke Scale.

\section{Keywords}

Mechanical thrombectomy, intravenous thrombolysis, multimodal computed tomography (CT), CT perfusion, fluid-attenuated inversion recovery magnetic resonance imaging/ diffusion weighted imaging (FLAIR/DWI) mismatch, diffusion perfusion mismatch

Disclosures: Horst Urbach, Elias Kellner and Karl Egger are shareholders of Veobrain $\mathrm{GmbH}$.

Review Process: Double-blind peer review. Compliance with Ethics: All procedures were followed in accordance with the responsible committee on human experimentation and with the Helsinki Declaration of 1975 and subsequent revisions. Informed consent was not obtained from the patients included in this special report, but no identifying information or images have been included.

Authorship: All named authors meet the criteria of the International Committee of Medical Journal Editors for authorship for this manuscript, take responsibility for the integrity of the work as a whole and have given final approval for the version to be published.

Received: 22 January 2019

Accepted: 16 April 2019

Citation: European Neurological Review. 2019;14(1):24-7 Corresponding Author: Horst Urbach, MD,

Department of Neuroradiology, University Medical Center Freiburg, Breisacher Str. 64, 79106 Freiburg, Germany. E: horst.urbach@uniklinik-freiburg.de

Support: No funding was received in

the publication of this article.
In 2018, two randomised controlled trials, DAWN (the Clinical Mismatch in the Triage of Wake up and Late Presenting Strokes Undergoing Neurointervention with Trevo Thrombectomy Procedure) and DEFUSE 3 (Endovascular Therapy Following Imaging Evaluation for Ischaemic Stroke 3), implemented computed tomography (CT) and magnetic resonance imaging (MRI) in patients with stroke.1,2 These studies showed the benefit of mechanical thrombectomy in patients with large vessel occlusions (LVO) presenting within 6-24 hours after symptom onset and having substantial tissue at risk. ${ }^{1,2}$ Another MRI-based randomised controlled trial, WAKE-UP (Efficacy and Safety of MRI-based Thrombolysis in Wake-up Stroke), showed the benefit of intravenous (IV) thrombolysis in patients with wake-up stroke. ${ }^{3,4}$ Do these study findings mean that a patient with LVO or wake-up stroke must be studied with MRI? Additionally, how does that concur with the fact that $>95 \%$ of stroke centres perform multimodal CT imaging in patients with acute stroke? Must these stroke centres now provide both CT and MRI capacities to investigate stroke patients? In order to answer these questions, it is necessary to take a closer look at the rationale and the results of these studies with the aim of devising an imaging algorithm.

\section{Rationale of the extended mechanical thrombectomy studies DAWN and DEFUSE 3}

The concept of the DAWN and DEFUSE 3 studies was to acquire magnetic resonance angiography or CT angiography (CTA) to prove the presence of LVO, to acquire MRI diffusion-weighted imaging (DWI) or CT perfusion (CTP) to depict the infarct core, and to acquire MRI perfusion-weighted imaging (PWI) or CTP to depict the tissue at risk. ${ }^{2}$ In DEFUSE 3, patients with occlusion of the internal carotid artery or occlusion of the first segment of the middle cerebral artery (M1) and a so-called target mismatch profile (ischaemic core volume $<70 \mathrm{~mL}$, mismatch ratio $>1.8$ and mismatch volume $>15 \mathrm{~mL}$ ) were included. The infarct core was defined as tissue with a relative cerebral blood flow $<30 \%$ compared with the contralateral hemisphere. This value was derived from the data of 103 patients, in which DWI-MRI was acquired in a mean of 36 minutes after CTP. ${ }^{5}$ Using this measure, the infarct core is not overestimated and smaller than on DWI. ${ }^{6}$

The tissue at risk in DAWN and DEFUSE 3 was defined as having a time to maximum of the residue function (Tmax) prolongation $>6$ seconds. Mismatch volume (tissue at risk - infarct core volumes) was determined in real-time with specific automated software (RAPID software, ischemaView, Inc., Menlo Park, California, USA).? This software is only available in some stroke centres in Europe, and although other software tools have achieved CE approval in the meantime, many stroke specialists treat patients within the 6-24-hour window as they would those within the 6-hour window. It means they do not consider CT or MRI perfusion but base their decision on the ASPECTS score. The infarct cores - tissue at risk maps presented in Figures 1-4 were calculated with the mRay-VEOcore software tool (www.veobrain.com). 
Table 1: Outcome of randomised controlled trials of mechanical thrombectomy with stent-retrievers versus best medical therapy

\begin{tabular}{|c|c|c|c|}
\hline Study & MT+BMM: mRS 0-2 & BMM: mRS 0-2 & OR (95\% Cl) \\
\hline MR CLEAN ${ }^{20}$ & 76/233 (33\%) & 51/267 (19\%) & 2.05 (1.36-3.09) \\
\hline EXTEND $\mid \mathrm{A}^{21}$ & 25/35 (71\%) & 14/35 (40\%) & 3.75 (1.78-10.17) \\
\hline ESCAPE $^{22}$ & 87/164 (53\%) & 43/147 (29\%) & $2.73(1.71-4.37)$ \\
\hline SWIFT PRIME ${ }^{23}$ & 45/103 (44\%) & 29/103 (28\%) & $2.75(1.53-4.94)$ \\
\hline REVASCAT $^{24}$ & $59 / 98$ (60\%) & 33/93 (35\%) & $1.98(1.11-1.53)$ \\
\hline THRACE $^{25}$ & 106/200 (53\%) & 85/202 (42\%) & $1.55(1.05-2.30)$ \\
\hline THERAPY ${ }^{26}$ & 19/50 (38\%) & $14 / 46$ (30\%) & $1.40(0.60-3.27)$ \\
\hline PISTE $^{27}$ & 17/33 (52\%) & $12 / 30$ (40\%) & $1.53(0.58-4.01)$ \\
\hline $\mathrm{EASI}^{28}$ & 19/35 (54\%) & 14/32 (44\%) & $2.03(1.68-2.46)$ \\
\hline DAWN $^{1}$ & $52 / 107$ (49\%) & 13/99 (13\%) & $2.10(1.10-3.00)^{*}$ \\
\hline DEFUSE $3^{2}$ & 41/92 (45\%) & 15/90 (17\%) & $2.67-(1.60-4.48)$ \\
\hline
\end{tabular}

*Good outcome had been defined as mRS of 0-2.

$B M M=$ best medical therapy; $\mathrm{Cl}=$ confidence interval; $m R S=$ modified Rankin score; $M T=$ mechanical thrombectomy; OR = odds ratio.

Figure 1: Diffusion-weighted imaging and perfusion magnetic resonance imaging of a patient with stroke and no mismatch

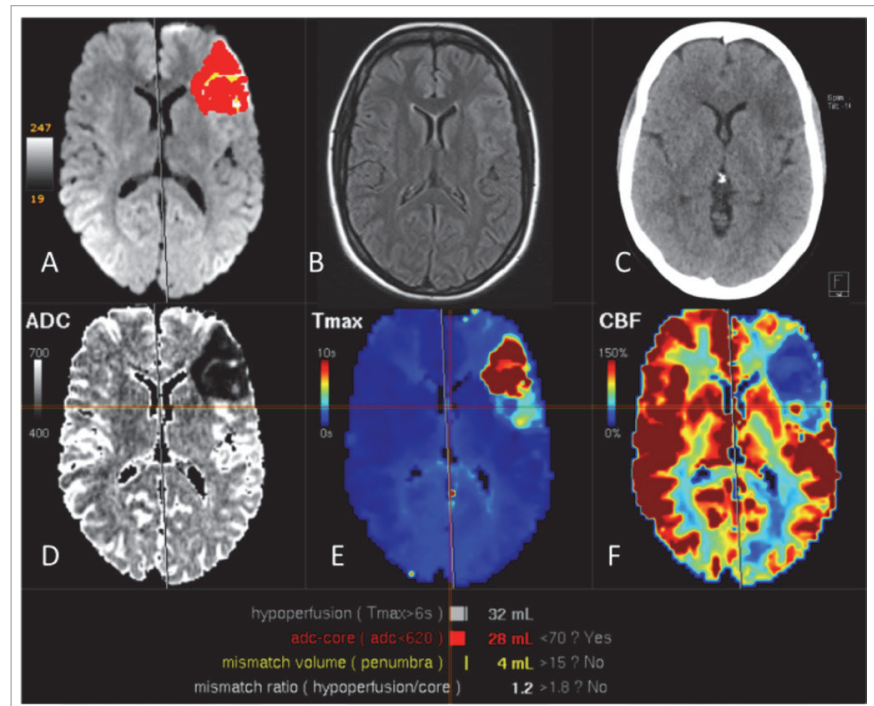

(A) Diffusion-weighted imaging with superimposed infarct core in red and tissue at risk in yellow; (B) Although the infarct is (clearly) visible on FLAIR MRI, the patient was treated with IV thrombolysis; (C) Follow-up CT after 1 day shows the infarct extension identical to the area in A; (D) ADC map; (E) Tmax map; (F) cerebral blood flow map.

$A D C=$ apparent diffusion coefficient; $C T=$ computed tomography; FLAIR MRI = fluidattenuated inversion recovery magnetic resonance imaging; IV = intravenous; Tmax = time to maximum of the residue function.

\section{Rationale of the WAKE-UP study}

If a patient wakes up with a stroke the time of onset cannot be determined. In the WAKE-UP study, this onset was estimated via DWI and fluid-attenuated inversion recovery (FLAIR) MRI. An infarct which is clearly visible on FLAIR is likely to be older than 4.5 hours and those patients are not considered suitable for IV thrombolysis. ${ }^{3}$ However, it is difficult to define what one might consider a clearly FLAIR-visible infarct. This has been shown in a post-hoc analysis in which 5\% of patients treated with IV thrombolysis were considered to have a FLAIR-visible infarct (Figure 1). ${ }^{4}$
Figure 2: Distal left-sided anterior cerebral artery occlusion in an 82-year-old woman with hemiparesis, aphasia and neglect (NIHSS 9)

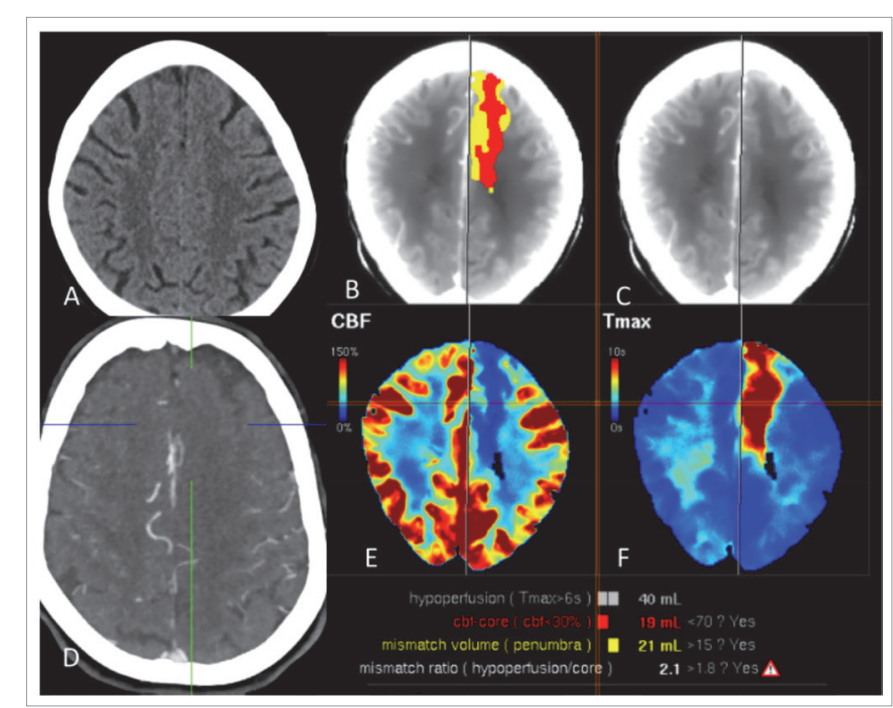

(A) Unenhanced CT 2 hours after symptom onset; (B and C) CTP with the tissue at risk superimposed in yellow (B), note that the red tissue is confined to the white matter and shows that collateralisation is worse than in the grey matter; (D) The thromboembolic occlusion was overlooked on CTA; (E) cerebral blood flow map; (F) Tmax map.

$C T=$ computed tomography; CTA = computed tomography angiography; CTP = computed tomography perfusion; NIHSS: National Institutes of Health Stroke Scale; Tmax = time to maximum of the residue function.

\section{Summary of the main results of DAWN, DEFUSE 3, WAKE-UP and other studies}

The results of these key clinical studies are illuminating but difficult to incorporate into one imaging concept. The DAWN and DEFUSE 3 studies showed that thrombectomy for acute/ischaemic stroke, given with standard care to patients within 6-24 hours or 6-16 hours of onset, had better disability/functional outcomes at 90 days than patients who received standard care alone ${ }^{1,2}$ (outcomes of key trials are summarised in Table 1). The WAKE-UP study showed that in patients with acute stroke of unknown time of onset, treatment with IV thrombolysis, which was guided by a mismatch between DWI and FLAIR in the brain region of the ischaemia, resulted in better functional outcomes but more intracranial haemorrhages than placebo at 90 days. ${ }^{3}$ The WAKE-UP study, however, also showed that the $36 \%$ of patients with National Institutes of Health Stroke Scale (NIHSS) 0-4 did not benefit from IV thrombolysis. ${ }^{4}$ In a retrospective analysis of databases, patients with a NIHSS between 5-10 showed benefit from IV thrombolysis, but also from mechanical thrombectomy if they had an LVO. ${ }^{8}$ Patients with a NIHSS $>10$ have a high probability (>65\%) of having an LVO and require DWI/PWI or CTP in order to prove that they have a small infarct core and substantial tissue at risk. ${ }^{9}$ The use of the NIHSS, however, is debated, and the cortical symptoms of neglect, aphasia alone or combined with motor symptoms, are more reliable indicators of an LVO than the NIHSS. ${ }^{10}$

\section{Proposal of an imaging algorithm}

If the NIHSS is to be used for selecting imaging modalities we propose the following imaging algorithm irrespective of the time window:

NIHSS 0-5: Unenhanced CT to exclude haemorrhage and CTA to exclude an LVO is sufficient. DWI is advantageous since it displays small lacunar or microembolic infarcts. AS CTP or PWI should not delay administration of IV thrombolysis, ${ }^{11}$ CTP can be acquired without quality-loss after CTA. 
Figure 3: A 67-year-old woman with a thromboembolic middle cerebral artery M1 segment occlusion due to atrial fibrillation (NIHSS 14)

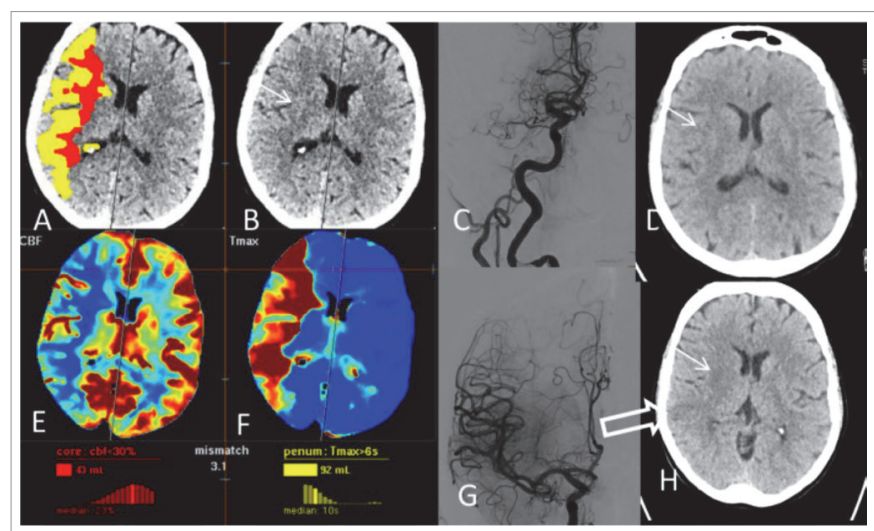

(A) CTP after 60 minutes shows tissue at risk of the cortical MCA territory; (C) thromboembolic MCA M1 segment occlusion due to atrial fibrillation. (G) The MCA was recanalised with three retrieval manoeuvres (Solitaire 6x40); (H) Follow-up CT at day 1 shows a small new cortical MCA infarct (open arrow) while the discrete hypodensity of the right lentiform nucleus was already visible on the initial CT (B, D, H: arrows). $C T=$ computed tomography; $C T P=$ computed tomography perfusion; $M 1=$ first segment of the middle cerebral artery; MCA = middle cerebral artery; NIHSS = National Institutes of Health Stroke Scale.

NIHSS 5-10: DWI/PWI or CT (CT/CTA/CTP) can be used interchangeably. Speed ("time is brain") and display of the LVO and the arterial access via the aortic arch are in favour of CTA/CTP. CTP better shows peripheral infarcts which are likely to be missed with CTA alone (Figure 2). It also better illustrates the tissue at risk volume in patients with $\mathrm{M} 2$ occlusions.

NIHSS > 10: Patients have a high likelihood of LVO and the need of mechanical thrombectomy. Speed, patient compliance, and display of LVO and arterial access via the aortic arch are in clear favour of CTA/CTP (Figures 3 and 4).

Current guidelines of the American Heart Association and the European Stroke Organisation - European Society of Minimally Invasive Neurological Therapy (ESO-ESMINT) do not recommend CTP or PWI within the first 6 hours following stroke onset.11,12 The rationale for this is that in a small subgroup of patients studied with $>70 \mathrm{~mL}$ infarct core volume on CTP, two of $25(8 \%)$ compared with none of 25 control patients achieved functional independence. ${ }^{12}$ We respect these recommendations and would rather treat a patient than excluding them. Those who would benefit and those who would not needs to be determined at an early stage. If these data are not acquired in the 0-6-hour window an opportunity is lost and it is not possible to understand subsequent changes in the brain tissue.

It is difficult to predict the functional outcome following successful recanalisation in an individual patient. This is because brain tissue dies
Figure 4: Computed tomography perfusion in an 83-year-old female with NIHSS 19

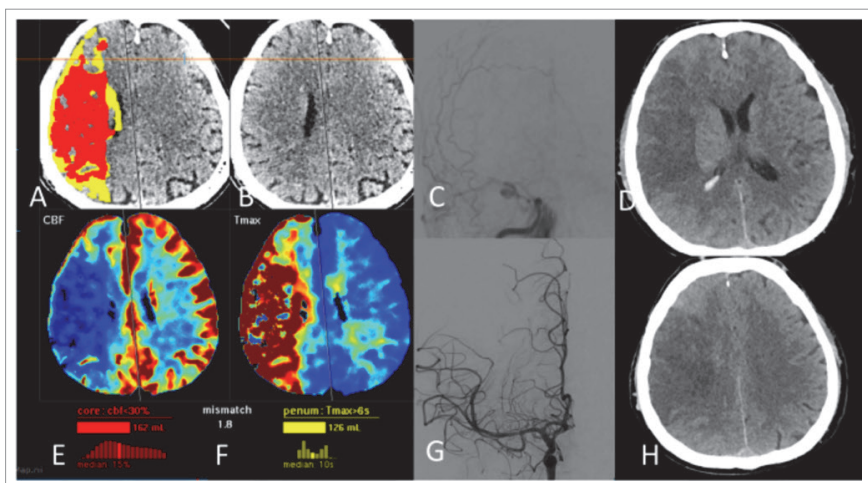

(A and B) Computed tomography perfusion 105 minutes after symptom onset shows a large infarct core and little tissue at risk; (G) Despite complete recanalisation of a carotid T occlusion (C) the patient had a large MCA infarct at day $1(H)$; (D) Note the initial unenhanced CT for comparison; (E) cerebral blood flow map; (F) Tmax map. CT = computed tomography; Tmax = time to maximum of the residue function; NIHSS = National Institutes of Health Stroke Scale.

at variable speeds in different patients. The following percentages may be used for outcome estimation provided that complete recanalisation is achieved. Less than $10 \%$ of patients with M1 or carotid T-occlusions already have large ischaemic core volumes $(>80 \mathrm{~mL}$ ) at their initial DWI examination within 3 hours. ${ }^{13}$ Patients with large ischaemic cores typically have a worse outcome than those with small ischaemic cores. ${ }^{14}$ The infarct core volume is reliably assessed with CTP but not with visual or eASPECTS analysis of unenhanced CT scans. ${ }^{15}$ The final infarct volume is strongly associated with the functional outcome.16

Around $20 \%$ of patients have large and severely hypoperfused tissue at risk (Tmax prolongation $>10$ seconds and mismatch volume $>100 \mathrm{~mL}$ = malignant imaging profile) with fast infarct growth rates from about $15 \mathrm{~mL} / \mathrm{h}$ to as high as $100 \mathrm{~mL} / \mathrm{h} \cdot .^{17,18}$ The vast majority of patients (likely $>70 \%$ ) fall into a category in which there is little or no infarct growth within the first day or the infarct core grows with a low rate of 3-10 $\mathrm{mL} / \mathrm{h} .{ }^{18,19}$ These patients clearly benefit from mechanical thrombectomy within the first 24 hours or even longer. The net benefit of this treatment compared with IV thrombolysis or supportive therapy (after 4.5 hours) is even larger in the late time window. ${ }^{18}$

In summary, an ideal imaging algorithm should consider the clinical deficit and, if known, the interval between onset of symptoms and imaging. In this context, multimodal acute stroke CT imaging can be used to indicate the suitability of mechanical thrombectomy and/or IV thrombolysis. Multimodal acute stroke CT imaging likely predicts the patient's outcome following recanalisation therapy better than the time interval between symptom onset and therapy.
Nogueira RG, Jadhav AP, Haussen DC, et al. Thrombectomy 6 to 24 hours after stroke with a mismatch between deficit and infarct. N Eng/ J Med. 2018;378:11-21.

2. Albers GW, Marks MP, Kemp S, et al. Thrombectomy for stroke at 6 to 16 hours with selection by perfusion imaging. Stroke at 6 to 16 hours with se

N Engl J Med. 2018,378.708-18. Thomalla G, Fiebach JB, Østergaard L, et al. A multicenter randomized, double-blind, placebo-controlled trial to test efficacy and safety of magnetic resonance imaging-base thrombolysis in wake-up stroke (WAKE-UP). Int I Stroke. 2014;9:829-36.

4. Thomalla G, Simonsen CZ, Boutitie F, et al. MRl-guided thrombolysis for stroke with unknown time of onset. N Engl J Med. 2018;379:611-22.

Cereda CW, Christensen S, Campbell BCV, et al. A benchmarking tool to evaluate computer tomography perfusion infarct core predictions against a DWI standard.
J Cereb Blood Flow Metab. 2016;36:1780-9.

Hoving IW Marquering HA Majoie CBLM et al Volumetric and spatial accuracy of computed tomegraphy perfusion estic and spatia accuracy of comp, Mad Stroke. 2018:49:2368-75.

Stroke. 2018;49:2368-75.
Straka M, Albers GW, Bammer R. Real-time diffusion-perfusion Straka M, Albers GW, Bammer R. Real-time amusion-perfusion mismatch analysis in acute stroke. J Magn Reson Imagin 2010;32:1024-37

8. Haussen DC, Lima FO, Bouslama M, et al. Thrombectomy versus medical management for large vessel occlusion strokes with minimal symptoms: an analysis from STOPStroke and GESTOR cohorts. J Neurointervent Surg. 2018;10:325-9.

Fischer U, Arnold M, Nedeltchev K, et al. NIHSS score and arteriographic findings in acute ischemic stroke. Stroke. 2005;36:2121-5.

10. Beume LA, Hieber M, Kaller CP, et al. Large vessel occlusion in acute stroke. Stroke. 2018:49:2323-9.
11. Powers WJ, Rabinstein AA, Ackerson T, et al. 2018 Guidelines for the early management of patients with acute ischemic strok earich American Heart Association/American Stroke Association. Stroke. 2018;49:e46-e110.

12. Turc $G$, Bhogal $P$. Fischer $U$, et al. European Stroke Organization (ESO)-European Society of Minimally Invasive Neurological Therapy (ESMINT) guidelines on mechanical thrombectomy in acute ischemic stroke. I Neurointerv Surg. 2019;11:535-8

13. Maurer CJ, Egger K, Dempfle AK, et al. Facing the time window in acute ischemic stroke: the infarct core. Clin Neuroradiol. 2016;26:153-8.

4. Campbell BCV, Majoie CBLM, Albers GW, et al. Penumbral imaging and functional outcome in patients with endovascular thrombectomy versus medical therapy: a 
meta-analysis of individual patient-level data. Lancet Neurol. 2018;18:46-55.

15. Demeestere J, Scheldeman L, Cornelissen SA, et al. Alberta Stroke Program early CT score versus computed tomographic perfusion to predict functional outcome after successful perfusion to predict functional outcome after successful
reperfusion in acute ischemic stroke. Stroke. 2018;49:2361-7.

16. Boers AMM, Jansen IGH, Beenen LFM, et al. Association of follow-up infarct volume with functional outcome in acute ischemic stroke: a pooled analysis of seven randomized trials. J Neurointerv Surg. 2018;10:1137-42.

17. Tsai JP, Mlynash M, Christensen S, et al. Time from imaging to endovascular reperfusion predicts outcome in acute stroke. Stroke. 2018;49:952-7.

18. Albers GW. Late window paradox. Stroke. 2018;49:768-71.

19. Guenego A, Mlynash M, Christensen S, et al. Hypoperfusion ratio predicts infarct growth during transfer for thrombectomy.
Ann Neurol. 2018;84:616-20

20. Berkhemer OA, Fransen PSS, Beumer D, et al. A randomized trial of intraarterial treatment for acute ischemic stroke. N Eng/ J Med. 2015;372:11-20.

21. Campbell BCV, Mitchell PJ, Kleinig TJ et al. Endovascular therapy for ischemic stroke with perfusion-imaging selection. N Engl J Med. 2015;372:1009-18.

22. Goyal M, Demchuk AM, Menon BK, et al. Randomized assessment of rapid endovascular treatment of ischemic stroke. N Engl J Med. 2015;372:1019-30.

23. Saver IL, Goyal M, Bonafe A, et al. Stent-retriever thrombectomy after intravenous t-PA vs. t-PA alone in stroke. N Engl J Med. 2015;372:2285-95.

24. Jovin TG, Chamorro A, Cobo E, et al. Thrombectomy within 8 hours after symptom onset in ischemic stroke. N Engl I Med. 2015;372:2296-306.
25. Bracard S, Ducrocq X, Mas JL, et al. Mechanical thrombectomy after intravenous alteplase versus alteplase alone after stroke (THRACE): a randomised controlled trial. Lancet Neurol. 2016;15:1138-47.

26. Mocco $J$, Zaidat $\mathrm{OO}$, von Kummer $\mathrm{R}$, et al. Aspiration thrombectomy after intravenous alteplase versus intravenous alteplase alone. Stroke. 2016;47:2331-8.

27. Muir KW, Ford GA, Messow C-M, et al. Endovascular therapy for acute ischaemic stroke: the pragmatic ischaemic stroke thrombectomy evaluation (PISTE) randomised, controlled trial. J Neurol Neurosurg Psychiatry. 2017;88:38-44.

28. Khoury NN, Darsaut TE, Ghostine J, et al. Endovascular thrombectomy and medical therapy versus medical therapy alone in acute stroke: a randomized care trial. J Neuroradiol. 2017;44:198-202. 